\title{
Clinical Case Reviews and Poster Sessions in Multiple Sclerosis Spasticity: Main Outcomes and Highlights
}

\author{
Maria Trojano $^{a}$ Elisabeth G. Celius ${ }^{b}$ Cecile Donzéc Guillermo Izquierdo ${ }^{d}$ \\ Francesco Patti ${ }^{e}$ Dieter Pöhlau ${ }^{f}$ \\ ${ }^{a}$ Department of Basic Medical Sciences, Neurosciences and Sense Organs, University of Bari, Bari, Italy; ${ }^{\mathrm{b}}$ Department \\ of Neurology, Oslo University Hospital, Ullevål, Oslo, Norway; ${ }^{\circ}$ Department of Neurorehabilitation, Groupement \\ Hospitalier de l'Institut Catholique de Lille, Lille, France; d Hospital Universitario Virgen Macarena, Seville, Spain; \\ e GF Ingrassia Department, Neuroscience Section, First Neurology Clinic, Multiple Sclerosis Centre Sicilia Region,

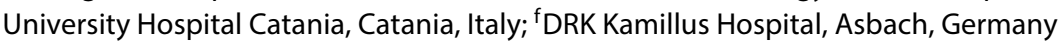

\section{Key Words}

Multiple sclerosis · Spasticity · Treatment-resistant ·

$\mathrm{THC}:$ CBD oromucosal spray

\begin{abstract}
Background: Individuals with multiple sclerosis (MS) spasticity present with a range of symptoms and disability levels that are frequently challenging to manage. Summary: Clinical case reviews in treatment-resistant MS spasticity were presented in five country-specific sessions conducted in parallel at the MS Experts Summit. Attendees at the Norwegian session discussed early response to new treatments for severe spasticity and highlighted the importance of titrating THC:CBD oromucosal spray (Sative ${ }^{\circledR}$ ) when adding it to baclofen. The French group focussed on MS symptoms and patient characteristics that interact with spasticity and agreed on a list of minimum ratings for diagnosis of MS spasticity symptoms. Attendees at the Spanish session concurred that THC:CBD oromucosal spray is effective and well tolerated as add-on therapy in treatment-resistant MS spasticity, particularly for pain, spasms and gait disturbances. The Italian group
\end{abstract}

discussed the use of add-on THC:CBD oromucosal spray and other possible combination therapies for treatment-resistant MS spasticity. Attendees at the German session highlighted the need to address trigger factors for MS spasticity to reduce the potential for impact on activities of daily living (ADL) and quality of life (QoL). Three innovative studies of MS spasticity from the poster session were selected for closer review. The MOVE $1 \mathrm{EU}$ epidemiological study indicated that, across western Europe, patients with MS spasticity continue to have unmet management needs. A literature review demonstrated that symptomatic relief of MS spasticity in patients who respond to THC:CBD oromucosal spray translates into sustainable improvements in ADL and QoL. Enriched-design studies of medications targeting the endocannabinoid system require careful interpretation due to possible pharmacodynamic 'priming', i.e. carry-over effects of successful active treatment during the enrichment phase. Key Messages: Sharing experiences of clinical practice, including experience with the use of THC:CBD oromucosal spray, may be useful to overcome some of the challenges in the overall management of patients with moderate to severe treatment-resistant MS spasticity.

\section{KARGER}

E-Mail karger@karger.com www.karger.com/ene (c) 2014 S. Karger AG, Basel

0014-3022/14/0721-0015\$39.50/0
Prof. M. Trojano

Department of Basic Medical Sciences, Neurosciences and Sense Organs University of Bari, Policlinico di Bari

Piazza Giulio Cesare 11, IT-70124 Bari (Italy)

E-Mail maria.troiano@uniba.it 


\section{Introduction}

\section{Clinical Case Reviews in MS Spasticity: Main}

Outcomes of Experiences from Around Europe

An ever-popular feature of the MS Experts Summit is the country-specific clinical case reviews. During these interactive sessions, various challenges faced by physicians when caring for patients with MS spasticity are explored through the presentation of case studies. The ensuing discussions offer attendees an opportunity to share personal experiences and learn from colleagues in their native language. Main discussion points and conclusions from each session are then summarized and shared among the wider audience. This year's event featured clinical case reviews from five EU countries, namely France, Germany, Italy, Norway and Spain, with each parallel session involving about 50 MS specialists ( $<10$ in the Norwegian session).

\section{Early Response to New Treatments in Patients with Resistant MS Spasticity}

Elisabeth Celius, Norway

E-Mail uxelgu@ous-hf.no

The Norwegian group agreed that patients not achieving adequate symptomatic relief of MS spasticity with baclofen should receive add-on treatment with THC:CBD oromucosal spray (table 1). Gradual titration is essential as most patients reach a threshold where dizziness develops and need to reduce the dose by 1-2 sprays/day. Most patients are satisfied with a dose of 4-7 sprays/day, but this can vary considerably.

The modified Ashworth spasticity scale (grading spasticity from $0-4$ ) and numerical rating scale (NRS, 0-10 visual analogue scale) are both used in Norway to measure spasticity. The NRS is considered to be more sensitive to change than the modified Ashworth scale. The group agreed that marked changes in spasticity severity are generally not observed within the first 3 months of use of THC:CBD oromucosal spray, whereas pain relief and functional improvement can be considerable. Side effects associated with THC:CBD oromucosal spray are generally as expected, although awareness of the possibility of new adverse events is always important; a rare event of severe diarrhea has been reported. Despite clinical benefits for pain, some patients decline the use of THC:CBD oromucosal spray because of dizziness. Overall, attendees considered THC:CBD oromucosal spray to be a favourable treatment option for management of MS spasticity.

\section{Diagnosis of MS Spasticity Symptoms}

Cecile Donzé, France

E-Mail Donze.Cecile@ghicl.net

In the French clinical cases session, discussion focussed on MS symptoms that interact with spasticity and patient characteristics
Table 1. Experience with THC:CBD oromucosal spray in Norway

\begin{tabular}{ll}
\hline Use & Usually as add-on therapy to baclofen \\
\hline Dose & $\begin{array}{l}\text { Titration is important } \\
\text { Individual dosing, average 4-7 sprays/day }\end{array}$ \\
\hline $\begin{array}{l}\text { Modified Ashworth } \\
\text { scale/Numerical }\end{array}$ & $\begin{array}{l}\text { A change in spasticity is not always seen } \\
\text { on the modified Ashworth scale } \\
\text { Rating Scale (NRS) }\end{array}$ \\
$\begin{array}{ll}\text { NRS better reflects the patient's experience } \\
\text { Effectiveness }\end{array}$ & $\begin{array}{l}\text { Within } 3 \text { months: less effect on spasticity, } \\
\text { greater effect on spasticity-related pain }\end{array}$ \\
\hline Side effects & $\begin{array}{l}\text { As expected } \\
\text { Some patients decline use (even with good } \\
\text { effect) due to side effects } \\
\text { New drug - new side effects possible (e.g. } \\
\text { diarrhea) }\end{array}$ \\
\hline
\end{tabular}

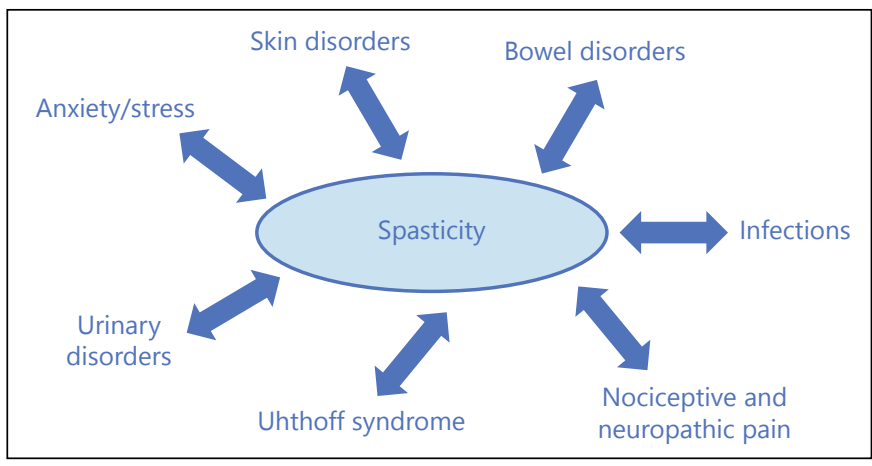

Fig. 1. Other symptoms of multiple sclerosis interact with spasticity.

that may worsen spasticity (fig. 1). The group agreed about the importance of recognizing symptoms that may influence spasticity which include, among others, musculoskeletal symptoms (e.g. tendinitis); orthopedic symptoms (e.g. osteoarthritis), urinary and bowel disorders; balance disorders (e.g. visual, oculomotricity, sensory or vestibular disturbances); fatigue/deconditioning (very common); Uhtoff syndrome (spasticity worsens when temperature is raised); and arm weakness.

The group agreed that minimum ratings for diagnosis of MS spasticity symptoms should include:

- A disability scale such as the Multiple Sclerosis Impact Scale (MSIS-29)

- NRS (0-10) / Modified Ashworth scale (0-4) for spasticity severity

- Visual analogue scale (VAS) to rate patients' subjective assessment of pain severity (both nocioceptive and neuropathic)

- Assessment of joint deformities; e.g. flexion deformity (flexum), equinovarus, valgus
16

Eur Neurol 2014;72(suppl 1):15-19 DOI: $10.1159 / 000367619$
Trojano/Celius/Donzé/Izquierdo/Patti/ Pöhlau 
- Balance assessments in static and dynamic conditions; eyes opened and closed, bi- and unipodal conditions

- Walk evaluations in ambulatory patients, bearing in mind the differences between the timed 25-foot walk test and the 2 - and 6-minute walk tests

- Irritative symptoms ratings (e.g. National Trauma Data Bank ISS score, urinary symptoms)

- Mobility ratings: motricity of iliopsoas, gluteus medius, quadriceps femoris and hamstrings, tibialis anterior and triceps surae

- Impact on activities of daily living (ADL) scale.

\section{Long-Term Management of MS Spasticity Symptoms}

Guillermo Izquierdo, Spain

E-Mail g.i.ayuso@gmail.com

In the Spanish clinical cases session, the group agreed that THC:CBD oromucosal spray is an effective and well tolerated addon therapy for MS spasticity and is especially useful for treating associated symptoms such as spasms and (particularly neuropathic) pain.

Attendees expressed the need for more objective assessments of treatment effects on gait disturbances and other MS spasticityrelated symptoms. For example, it was suggested that, in situations where space is adequate, use of the GAITRite walking system (a data-based analysis of gait) might overcome some of the criticisms associated with subjective assessments of mobility and spasticity. Investigations have shown a strong correlation ( 0.92$)$ between the GAITRite system and the timed 25-foot walk test. 'Before and after' video footage of a clinical case showed the patient clearly walking faster after 6 months' treatment with THC:CBD oromucosal spray. Objective assessment of the impact of symptomatic drug treatment on gait may be particularly important for patients receiving physiotherapy or other disability treatment.

\section{Treating Resistant MS Spasticity}

\section{Francesco Patti, Italy}

E-Mail patti@unict.it

In the Italian clinical cases session, attendees agreed unanimously that baclofen is the most common first-line treatment for MS spasticity in Italy. In the event of inadequate response to baclofen, recommendations are to add THC:CBD oromucosal spray, particularly for patients with more severe disease (e.g. EDSS >7.0) but also for those with a lower level of disability. Opinion on this latter point was divided, however, as many clinicians believe that patients with lower EDSS scores are better responders to THC:CBD oromucosal spray. Evidence to determine the best candidates for treatment of baclofen-resistant spasticity with THC:CBD oromucosal spray is currently insufficient and it is likely that both groups of patients may benefit from treatment.

Among other potential combinations of antispasticity medications, adding benzodiazepines to baclofen may be effective for spinal spasticity, while concomitant gabapentin may be effective for brain spasticity with marked neuropathic pain. Experience with use of intrathecal baclofen in Italy remains limited, with data available for $<300$ patients. The group agreed that it is appropriate to add pharmacotherapy to the physiotherapy regime for MS spasticity when the disability level is EDSS $\geq 4.0$.

In Italy, specialist MS centres tend to focus on less disabled patients (e.g. those with clinically isolated syndrome or relapsingremitting MS). Once disease-modifying therapy has been completed, symptomatic treatment-resistant patients are typically seen about once a year or less. Some of the Italian group considered that it would be useful to evaluate such patients every 3-6 months. Medications that provide symptomatic relief such as THC:CBD oromucosal spray and baclofen provide clinicians with additional options to manage their patients with MS spasticity.

\section{Quality of Life in MS Spasticity}

Dieter Pöhlau, Germany

E-Mail Dieter.Poehlau@Kamillus-Klinik.de

In the German clinical case reviews session the issue of quality of life (QoL) in patients with MS spasticity was addressed. In patients with resistant spasticity, associated symptoms such as pain, orthopaedic problems and bladder dysfunction tend to have the greatest negative impact on QoL. The group agreed on the importance of identifying and addressing trigger factors for spasticity and treating patients with physiotherapy and standard spasticity treatments, generally baclofen. Failing baclofen alone, the addition of THC:CBD oromucosal spray is recommended. For patients who experience 'breakthrough' spasticity in between normal dosing patterns, one or two additional sprays of THC:CBD oromucosal spray may provide relief. Patients must be adequately informed and trained regarding the use of THC:CBD oromucosal spray. Importantly, the availability of a treatment such as THC:CBD oromucosal spray that can combat symptoms effectively on an 'as needs' basis has a profound psychological impact on patients as it reduces the sense of helplessness which is a strong predictor of depression.

\section{Highlights of Poster Sessions in MS Spasticity}

Twenty posters presenting recent data were on display at the 2014 MS Experts Summit. Of these, three posters were selected by the chair to be reviewed in detail as they summarized relevant new data in MS spasticity research.

\section{MS Spasticity ‘Burden of Disease' in Europe}

The epidemiological burden of disease MOVE (MObility ImproVEment) 1 study from Germany reported that MS-related spasticity impaired patients' personal well-being and QoL, and that patients and physicians were largely dissatisfied with pharmacological options for spasticity [1]. Using a protocol similar to that of the German study, MOVE 1 was extended to other European countries; 300 
patients were recruited from 32 centers in seven countries: Belgium, Finland, France, Ireland, Norway, Poland and Portugal [2]. The MOVE 1 EU study combined 12-month retrospective chart documentation with cross-sectional questionnaires for physicians and patients at the time of enrolment. Most patients had secondary progressive MS (53.8\%) or relapsing remitting MS (32.3\%). Approximately $71 \%$ of patients were receiving antispasticity medication, most commonly baclofen or tizanidine. MS spasticity was moderate based on patients' self-reported scores of spasticity severity in the previous 24 hours (mean score of $5.1 \pm 2.6$ on the $0-10 \mathrm{NRS}$ ).

The most impairing symptoms of MS spasticity were muscle stiffness (77\% of patients) and mobility restrictions (69\%). MS spasticity restricted daily activities at least several times a week in about two-thirds of patients (as assessed by physicians and self-reported by patients using the EQ-5D) and, for the majority of patients, caused 'some' or 'moderate' problems in the subdomains of mobility, usual activities and pain/discomfort. Overall, 48\% of physicians and $34 \%$ of patients were at least partly dissatisfied with the effectiveness of available pharmacotherapy options for MS spasticity. The results of MOVE $1 \mathrm{EU}$ align with those of MOVE 1 Germany and highlight the need for optimizing the management and well-being of patients with MS spasticity across Europe.

\section{Impact of THC:CBD Oromucosal Spray on Daily Activities and Quality of Life in Patients with MS Spasticity}

In patients with MS, the presence of spasticity further restricts mobility and contributes to overall disability, often interfering with the ability to perform activities of daily living (ADL). Associated symptoms such as spasms, pain and sleep disturbances combine to impact negatively on QoL. Cross-sectional studies from Europe have indicated that advancing severity of MS spasticity correlates directly with worsening QoL $[1,3,4]$.

A literature review was undertaken to evaluate the potential of THC:CBD oromucosal spray to reduce the associated impact of MS spasticity on patients' ADL and QoL [5]. In randomised controlled trials and observational 'real life' studies conducted in patients with resistant MS-related spasticity, symptomatic relief of spasticity and related symptoms in responders to THC:CBD oromucosal spray translated into improvements in $\mathrm{ADL}$ and QoL as measured by the Barthel ADL index and MSQoL-54 instruments. Responder analyses consis- tently demonstrated a more pronounced effect in patients with an initial response $(\geq 20 \%$ NRS improvement) or clinically relevant response ( $\geq 30 \%$ NRS improvement) to THC:CBD oromucosal spray, thus substantiating the 'trial of therapy' approach in patients receiving the medication for the first time. Improvements in daily activities were maintained in patients receiving long-term treatment with THC:CBD oromucosal spray and were supported by caregiver perceptions of improvements in activities generally requiring assistance such as washing, dressing and transferring (e.g. from wheelchair to bed).

\section{Placebo Effects in An Enriched-Design Study of THC:CBD Oromucosal Spray for MS Spasticity}

Enriched-design studies are useful to identify responders to treatment while limiting exposure of a substance to individuals most likely to benefit. However, enriched design studies may be open to interpretation biases if changes in the trial period are not considered. To better understand the placebo effect observed during the randomized phase of an enriched-design study of THC:CBD oromucosal spray (i.e. after the 4-week trial of therapy phase) [6], the magnitude of the placebo effect was examined in three standard studies in which parallel groups of patients with treatment-resistant MS spasticity had been randomized to THC:CBD oromucosal spray or placebo from the start of treatment [7]. A pooled analysis of these studies showed a greater degree of separation between active treatment and placebo. Application of this same degree of separation to the results of the enriched-design study yielded an estimated mean therapeutic gain for THC:CBD oromucosal spray over placebo of 1.27 points $(20 \%)$ from the NRS score at screening. The magnitude of the placebo effect in the post-enrichment phase of an enriched-design study may be influenced by carry-over effects of successful active treatment during the enrichment phase (i.e. pharmacodynamic 'priming') and may be particularly relevant for medications targeting the endocannabinoid system. Careful interpretation of enriched-design studies is therefore recommended.

\section{Disclosures/Conflict of Interest}

MT has received personal compensation as a consultant or speaker from Biogen Idec, Novartis, Merck Serono and Almirall. MT has received research grants paid to her institution from Merck Serono, Biogen Idec, Novartis and Teva.
18

Eur Neurol 2014;72(suppl 1):15-19 DOI: $10.1159 / 000367619$
Trojano/Celius/Donzé/Izquierdo/Patti/ Pöhlau 
EGC has received honoraria and/or research grants from Almirall SA, Biogen Idec, Genzyme, Novartis and Teva.

$\mathrm{CD}$ has received honoraria, research grants or speaker's fees from Almirall SA, Biogen Idec, Coloplast, Merck, Novartis and Teva.

GI has received honoraria from Almirall SA, Bayer, Biogen Idec, Merck Serono, Novartis, Sanofi Genzyme and Teva.

FP has undertaken advisory activities for Almirall, Bayer, Biogen Idec Italy, Merck Serono, Sanofi Genzyme and Novartis. He has received research support from FISM and MIUR; personal compensation for speaking activities from Bayer, Biogen Idec Italy, Merck Serono, Novartis and TEVA; and travel grants from Bayer, Biogen Idec Italy, Merck Serono, Novartis and Sanofi Genzyme.

DP has received honoraria as a speaker from Almirall, BiogenIdec, Novartis, Teva and Sanofi Aventis.

Writing assistance was provided by Content Ed Net (Madrid, Spain), with funding from Laboratorios Almirall SA (Barcelona, Spain).

\section{References}

1 Flachenecker P, Henze T, Zettl UK: Spasticity in patients with multiple sclerosis-clinical characteristics, treatment and quality of life. Acta Neurol Scand 2013;129:154-162.

2 Vermersch P: MObility ImproVEment with spasticity in multiple sclerosis in Europe: the MOVE 1 EU study. Neurodegener Dis Manag 2014 [submitted].

-3 Arroyo R, Massana M, Vila C: Correlation between spasticity and quality of life in patients with multiple sclerosis: the CANDLE study. Int J Neurosci 2013;123:850-858.
4 Svensson J, Borg S, Nilsson P: Costs and quality of life in multiple sclerosis patients with spasticity. Acta Neurol. Scand. 2014;129:1320.

5 Arroyo R, Vila C, Dechant K: Impact of Sativex on quality of life and activities of daily living in patients with multiple sclerosis spasticity. J Comp Eff Res 2014 [accepted].
6 Novotna A, Mares J, Ratcliffe S, Novakova I, Vachova M, Zapletalova O, Gasperini C, Pozzilli C, Cefaro L, Comi G, Rossi P, Ambler Z, Stelmasiak Z, Erdmann A, Montalban X, Klimek A, Davies P; Sativex Spasticity Study Group: A randomized, double-blind, placebo-controlled, parallel-group, enriched-design study of nabiximols* (Sativex $\left({ }^{\circledR}\right)$ ), as add-on therapy, in subjects with refractory spasticity caused by multiple sclerosis. Eur J Neurol 2011;18:1122-1131.

7 Di Marzo V, Centonze D: Placebo effects in a multiple sclerosis spasticity enriched clinical trial with the oromucosal cannabinoid spray (THC/CBD, Sativex ${ }^{\circledR}$ ). Eur J Neurol 2014 [submitted]. 